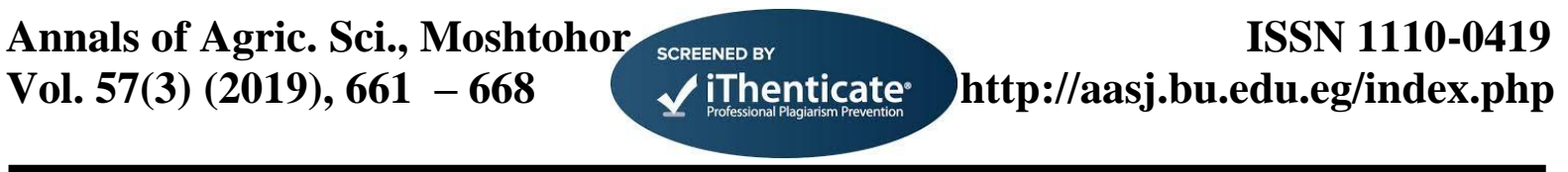

\title{
Using some fiber Properties to Predict Strength and Regularity of Combed Yarns for Some Egyptian Cotton Varieties
}

\author{
Hamed S. A. Ahmed and A. A. Hassan \\ Cotton Res. Inst., Agric. Res. Cent., Giza, Egypt \\ Corresponding author: dr.hamedsayed@yahoo.com
}

\begin{abstract}
The present investigations was carried out to determine prediction equation and the relative contribution of five fiber properties to estimate yarn strength, neps and evenness on count 80,100 and 120 for Extra-long (extra fine) and Extra-long stable of Egyptian cotton varieties under combed ring spinning systems.

The full model regression and stepwise multiple linear regression model were used to satisfy the target of work. The results of the supposed models of regression differed according to the Extra-long (extra fine) and extra-long of Egyptian cotton varieties and also the applying yarn counts.

Generally, under combed ring spinning system, Upper half mean Length was the most effective fiber properties to predict yarn properties for the Extra-long Extra fine, Extra-long stable on counts 80100 and 120 while, fiber strength and micronaire were the most important fiber traits contributing to yarn properties using the Extra-long (extra fine) stable on count 120 in Egyptian cotton varieties. The Fiber diameter was the greatest influence on the studied yarn properties for the Extra-long staple on count 120while, Fiber Diameter ranked the first in fiber properties that used to predict the yarn strength on count8os for the Extra-long stable in Egyptian cotton varieties.

All the supposed models of regression were significant and reflected large part of the variation of studied yarn properties expressed as high values of $\mathrm{R}^{\mathbf{2}}$ and near values of the corresponding adjusted $\mathrm{R}^{\mathbf{2}}$ indicating the validity and goodness of fit for these models.
\end{abstract}

Keywords: yarn counts, yarn strength, yarn neps, yarn evenness, combed ring spinning system and fiber properties.

\section{Introduction}

The combing process is normally used to produce smoother, finer, stronger and more uniform yarns. Therefore, combing is commonly confined to high grade, long staple natural fibers. In recent years, combing has been utilized for upgrading the quality of medium staple fibers. In addition, a yarn made of combed cotton needs less twist than a carded yarn. However, these quality improvements are obtained at the cost of additional expenditure on machines, floorspace and personnel, together with a loss of raw material. Yarn production coast is increased by something under $1 / \mathrm{Kg}$ of yarn (depending on the intensity of combing).

To improve the yarn quality, the comber must perform the following operations:

$>$ Elimination of precisely pre-determined quantity of short fibers

$>$ Elimination of the remaining impurities;

$>$ Elimination of a large proportion (not all) of the neps in the fiber material

$>$ Formation of a sliver having maximum possible evenness

$>$ producing of more straight and parallel fibers.

Fiber physical and yarn strength properties play an exceptionally important role as principle determinants of textile product quality. Yarn nep count and evenness are the most important features that influence the appearance of yarns and consequently the quality of the end product, especially in fine yarns which are needed in manufacturing fine and luxurious end products. Information concerning the relative contribution of fiber properties to yarn strength, yarn nep count, and evenness in different types of spinning system for various categories of cotton (Extra-long stable and long stable), is one of the important targets for each of cotton breeder to explore yarn quality of his large number of new selections also for the spinner in order to choose cottons that are best suited to the manufacture of specific end product.

Many authors used the full model regression as an important statistical procedure to estimate the relative contribution of the fiber properties, as independent variables, in the total variation of yarn properties as dependent variable. Also, the step wise multiple linear regressions were used to construct a regression equation that includes fiber properties accounting for the most variation of yarn properties.

Marth et al. (1952) reported that micronaire value was an excellent index of the number of neps expected in card web. Fiori et al. (1954) concluded put that fiber strength does not significantly affect the evenness of silver. Grosberg (1956) obtained a relationship between yarn unevenness coefficient of variation and the means of fiber length and diameter. Leitgeb and Wakeham (1956) pointed that neps increased with increasing fiber fineness. Phillip 
(1957) found that fiber fineness and maturity were related tonippiness. Balasubramanian and Lyengar (1961) reported that fiber length variation coefficient contributed more toyarn irregularity.Louis et al. (1961) concluded from their study on pima cottons that nep count is influenced by fiber elongation. Louis et al. (1968) stated that 5o\% span length was better than $2.5 \%$ span length to explain effect of fiber length on yarn strength. Rusca (197o) stated that neps rapidly decreased with increase in micronaire reading while yarn irregularity slightly decreased with either extra-long fine or extra-coarsemicronaire reading. Amin (1971) found that fiber strength and elongation were more effective than micronaire value with regard to their contribution to yarn strength. Garawain (1971) showed that yarn added unevenness was not highly related to micronaire reading. Sallouma et al. (1973) and AboulFadl (1976) found a strong association between micronaire value and the number of neps in yarn.

El-Ghawas et al. (1978) found that, within a variety, micronaire value and 5o\% Span length were negatively correlated with yarn evenness. The net effect of $50 \%$ Span length on yarn evenness was more pronounced than that of micronaire value. They also added that, on the basis of the relative net effect on pertinent fiber properties on yarn nippiness, micronaire value ranked first, followed by fiber length. Ahmed et al. (1984) Stated that, micronaire value exerted the greatest influence, wet hear directly or indirectly, upon yarn nippiness, followed by fiber length which appeared amoderate effect. Mansour (1984) found that the three fiber properties span length $(2.5 \%)$, fiber strength and micronaire value)were the most influential on evenness in spite of their rank which differed from one variety to.

Another and within the some variety from count to count. Working on the Egyptian Extra-long staple variety Giza 45, Seif (1984) found that fiber strength and micronaire values their interaction arose as the prominent factors contributing to yarn strength different counts and twist. Hegabet al. (1985) found that fiber strength, fiber length their interaction were the contributors to yarn strength in variety of Giza 70. Abdel-Fattah (1988) found that 2.5\% span length was the most contributors to yarn strength. Fares et al. (2010) indicated that fiber strength and short fiber content were the most important contributing cotton fiber properties in carded ring skein strength under four counts of spinning.

Ahmed and Hassan (2016) Found that the Micronaire reading was the most important causative factor inducing neps in cotton yarns. In fact, Micronaire reading exerted influence both directly and indirectlyup on neps count. Fiber length showed amoderate effect, while fiber stiffness revealed the lowest effect on yarn neps count compared with the other two factors.

Hassan and Ibrahim (2018). Indicated that fiber length, fiber strength and micronaire value had the greatest influenceon yarn strength, evenness C.v.\% and number of neps for the extra-long and long staple cotton varieties. All calculated models of regression were significant and reflected large part of the variation of studied yarn properties expressed as high values of $\mathrm{R} 2$.

The present investigation was carried out to the present investigations were carried out to determine prediction equation and determine the relative importance of fiber properties to explain the variation of yarn strength, yarn nep count and evenness on counts 80s, 100s and 120s using combed ring spinning systems for Extra-long extra fine, Extralong staple in Egyptian cotton varieties.

\section{Materials and methods}

The present study was carried out on twostaplesof Egyptian cotton varieties, that are, Extra-long(extra fine) staple (Giza 45, Giza 87 and Giza 93) and Extra-long staple (Giza 88, Giza 92, and Giza 70) during 2016 season at laboratories of Cotton Technology Division of the Cotton Research Institute. Cotton samples were obtained from the commercial cotton samples of 2016 crop that belong to Cotton Maintaince Research Section. Cotton samples were spun into Combed Ring Spinning systems at one count $(80,100$ and120noil $18 \%)$ with 4.3 twist factor Lab labor

Three yarn quality properties were used as dependent variables in the current study that are, Y1strength, Y2neps count and Y3evenness (c.v\%)was measured on the Good Brand Lea Tester to determine the lea strength in pounds A.S.T.M. (1967), D1578. Nep count and yarn evenness (c.v. $\%$ ) were measured by Uster tester 3 according to the standard methods testing of textile material A.S.T.M. (1986), D- 1425- 60 T).

Data were recorded for five fiber properties as independent variables vise:

X1 Upper half mean Length ( $\mathrm{mm})$

X2Fiber strength (g/tex)

X3 Micronaire

X4Short fiber content

X5Fiber Diameter

Fiber properties were tested according to the following methods:

H.V.I Instrument System was used according to A.S.T.M (D-4603-86-1776-98) to determine Upper Half Mean Length (UHM) by (mm.), fiber Strength by(g/tex), Micronaire, and Short fiber content was used according to the A.S.T.M.(D-1440-65).

\section{Statistical analysis}

The full model regression and step wise multiple linear regression were used to evaluate the relative contribution of the aforementioned fiber properties $(\mathbf{X 1}, \mathbf{X 2}, \ldots . . \mathrm{X5})$ as explanatory variables on each of three qualityyarn characters Y1 strength, Y2neps 
count and Y3evenness (c.v\%) as dependent variables.

Full model regression was applied as outlined by Draper and Smith (1981) to predict the quality yarn properties using fiber properties and also to estimate the relative importance of the fiber properties expressed as coefficient of determination $\left(\mathrm{R}^{2}\right.$ value) in the total variation of yarn characters.

Stepwise multiple linear regression analysis was used to determine what of the fiber properties that accounted for the majority of the total variation of yarn quality properties as described by Draper and Smith (1981).

\section{Results and discussion}

Data in Table 1 indicate that the correlation among fiber properties and yarn, strength, neps and evenness (c.v\%) within each yarn counts $(80,100$ and 120) over theExtra-long (extra Fine) and Extra-long stapleusing combed ring spinning system.

Thepositive highly significant correlation was found between yarn strength on count 8 os and each of Upper half mean length (mm), fiber strength (g/tex),micronaire and short fiber content for Extralong (extra fine staple $(0.91,0.50,0.71$ and 0.38 respectively). In addition positive highly significant correlation was detected between yarn strength on count100 and upper half mean length $(\mathrm{mm})$,micronaire, short fiber content and fiber diameter for extra-long (extra fine) staple (0.72, $0.42,0.45$ and 0.36 respectively).Moreover, positive highly significant correlation was detected between yarn strength on count 120 and upper half mean length $(\mathrm{mm})$, fiber strength $(\mathrm{g} / \mathrm{tex})$ micronaire , short fiber content and fiber diameter for extra-long extra fine staple $(0.98,0.80,0.53,0.48$ and 0.62 respectively). These results in general are agreement with thoseobtained by Sallouma et al. (1973),Nawar etal. (1990), Raoof (1995) and Abdel-Salam(1999).

on the other hand, negative highly significant correlation was found between neps on count 8o's and each of upper half mean length (m.m), fiber strength (g/tex), short fiber content and fiber diameter for extra-long extra fine staple $(-0.84,-$ $0.62,-0.73$ and -0.92 respectivly). Highly negative correlation was found among neps on count 1oo's and each of upper half mean length (m.m), fiber strength (g/tex), short fiber content and fiber diameter for extra-longextra fine staple $(-0.67,-0.76$,0.57 and -0.87) respectively. Moreover negative highly significant correlation was found between neps on count 12o's and each of upper half mean length (m.m), fiber strength (g/tex), short fiber content and fiber diameter for extra-long -extra fine staple $(0.61,-0.77,-0.52$ and -0.83$)$. In addition positive highly significant correlation was detected between evenness (c.v\%) on counts 80,100 and each of upper half mean length (m.m), short fiber content and fiber diameter for Extra-long -extra fine staple .

Table 1. Correlation coefficient between fiber and yarn properties within each of three yarncounts $(80,100$ and 120) in two staples of Egyptian cotton.

\begin{tabular}{|c|c|c|c|c|c|c|}
\hline \multirow{2}{*}{ counts } & \multirow{2}{*}{ Yarn properties } & \multicolumn{5}{|c|}{ Fiber properties } \\
\hline & & $\left(\mathrm{X}_{1}\right)$ & $\left(\mathrm{X}_{2}\right)$ & $\left(\mathrm{X}_{3}\right)$ & $\left(\mathrm{X}_{4}\right)$ & $\left(\mathrm{X}_{5}\right)$ \\
\hline \multicolumn{7}{|c|}{ Extra-long (Extra fine) staple } \\
\hline \multirow{4}{*}{80} & $\left(\mathrm{Y}_{1}\right)$ Strength & $0.91 * *$ & $0.50 * *$ & $0.71 * *$ & $0.38 * *$ & 0.34 \\
\hline & $\left(\mathrm{Y}_{2}\right) \mathrm{Neps}$ & $-0.84 * *$ & $-0.62 * *$ & -0.05 & $-0.73^{* *}$ & $-0.92 * *$ \\
\hline & $\left(\mathrm{Y}_{3}\right)$ Evenness (c.v\%) & $0.73 * *$ & 0.26 & -0.08 & $0.92 * *$ & $0.85 * *$ \\
\hline & $\left(\mathrm{Y}_{1}\right)$ Strength & $0.72 * *$ & 0.30 & $0.42 * *$ & $0.45^{* *}$ & $0.36 * *$ \\
\hline \multirow[t]{2}{*}{100} & $\left(\mathrm{Y}_{2}\right) \mathrm{Neps}$ & $-0.67 * *$ & $-0.76 * *$ & 0.06 & $-0.57 * *$ & $-0.87 * *$ \\
\hline & $\left(\mathrm{Y}_{3}\right)$ Evenness (c.v\%) & $0.47 * *$ & 0.33 & $-0.44 * *$ & $0.83 * *$ & $0.96 * *$ \\
\hline \multirow{3}{*}{120} & $\left(\mathrm{Y}_{1}\right)$ Strength & $0.98 * *$ & $0.80 * *$ & $0.53 * *$ & $0.48 * *$ & $0.62 * *$ \\
\hline & $\left(\mathrm{Y}_{2}\right) \mathrm{Neps}$ & $-0.61 * *$ & $-0.77 * *$ & 0.08 & $-0.52 * *$ & $-0.83 * *$ \\
\hline & $\left(\mathrm{Y}_{3}\right)$ Evenness $(\mathrm{c} . \mathrm{v} \%)$ & $-0.49 * *$ & $-0.76 * *$ & $0.09 * *$ & $-0.36 *$ & $-0.72 * *$ \\
\hline \multicolumn{7}{|c|}{ Extra-long staple } \\
\hline \multirow{3}{*}{80} & $\left(\mathrm{Y}_{1}\right)$ Strength & $0.30 * *$ & $0.59 * *$ & $0.39 * *$ & $-0.58 * *$ & $-0.65 * *$ \\
\hline & $\left(\mathrm{Y}_{2}\right) \mathrm{Neps}$ & $-0.37 * *$ & $-0.90 * *$ & $-0.40 * *$ & $0.29 * *$ & $0.95 * *$ \\
\hline & $\left(\mathrm{Y}_{3}\right)$ Evenness (c.v\%) & $-0.68 * *$ & $-0.62 * *$ & $-0.69 * *$ & $0.26 * *$ & $0.69 * *$ \\
\hline \multirow{3}{*}{100} & $\left(\mathrm{Y}_{1}\right)$ Strength & $0.93 * *$ & $0.40 * *$ & $0.94 * *$ & -0.01 & $-0.51 * *$ \\
\hline & $\left(\mathrm{Y}_{2}\right) \mathrm{Neps}$ & $-0.20 * *$ & $-0.84 * *$ & $-0.26^{* *}$ & $0.49 * *$ & $0.91 * *$ \\
\hline & $\left(\mathrm{Y}_{3}\right)$ Evenness (c.v\%) & $-0.77 * *$ & $-60 * *$ & $-0.82 * *$ & 0.17 & $-0.71 * *$ \\
\hline \multirow{3}{*}{120} & $\left(\mathrm{Y}_{1}\right)$ Strength & 0.05 & $0.88 * *$ & -0.01 & 0.17 & $-0.84 * *$ \\
\hline & $\left(\mathrm{Y}_{2}\right) \mathrm{Neps}$ & $-0.50 * *$ & $-0.91 * *$ & $-0.48 * *$ & 0.12 & $0.91 * *$ \\
\hline & $\left(\mathrm{Y}_{3}\right)$ Evenness (c.v\%) & $-0.58 * *$ & $-0.80 * *$ & $-0.57 * *$ & $0.23 * *$ & $0.87 * *$ \\
\hline
\end{tabular}

$\mathrm{X} 1=$ (U.H.M), Upper half mean Length (m.m), X2= (F. stre.), Fiber strength (g/tex), X3(Mic.), Micronaire, X 4= (S.F.C), Short fiber content and X5= (F.d), Fiber diameter

Positive highly significant correlation was measured between yarn strength on count 8o's 100 andupper half mean length (m.m) fiber strength (g/tex) andmicronaire for extra-long staple. while, 
negative highly significant correlation was found between yarn strength on counts 80 "s and short fiber content, fiber diameter for extra-long staple. Highly significant negative correlation was found among neps on counts 8o's,1oo's and 12o's and each of upper half mean length (m.m) fiber strength and micronaire for extra-long staplewhile, positive highly significant correlation was detected between neps on counts 8o's, 1oo's and 12o's and each of short fiber content, fiber diameter for extra-long staple. Highly significant negative correlation was found among evenness (c.v\%) on counts 80100 and 120 and each of upper half mean length (m.m), fiber strength (g/tex) and micronaire for extra-long staple -while positive highly significant correlation was detected between evenness (c.v\%)on counts 8o's 12o's and each of short fiber content fiber diameter for extralong staple, and Significant and highly significant at 0.05 and 0.01 probability Respectively Sagbaş and Erol (2004).Strumiłloet al., (2007).

The results of multiple linear regression analysis between each oneof threeyarn properties on counts 80,100 and 120 as dependent variable and five fiber properties as explanatory variables under combed ring spinning system of Extra-longExtra Fin stable Egyptian cotton varieties are presented in table 2.The results revealed that the supposed multiple regression models were significantly explained the most variability of the theeyarn properties on counts 8os, 1oos, and 12o,s of Extra-long Extra Fin stable Egyptian cotton varieties. Statistically, goodness of fit was satisfied for the nine supposed models where the coefficients of determination $\left(\mathrm{R}^{2} \%\right)$ ranged from 70.7 to 98.6 indicating that the most yarn properties variation was attributed to the tested fiber properties. The residuals content $\left(1-\mathrm{R}^{2} \%\right)$ may by returned to some errors during measuring the fiber and yarn properties, some fiber properties were not into account under the current investigation and / orun known variation (randomerror).one the other hand, the values of adjusted $\mathrm{R}^{2}$ werevery close to their corresponding $\mathrm{R}^{2}$ values giving evidence on the goodness of fit for the supposed models. Similar trend of results was obtained by Krifa and Ethridge(2003), Krifa and Ethridge(2006), Fares et al.(2010) and Hager et al., (2011).

Table 2. Regression Coefficient to predict fiber and yarn properties within each of three yarncounts(80, 100 and 120) in two staples of Egyptian cotton under combed ring spinning system.

\begin{tabular}{|c|c|c|c|c|c|c|c|c|c|}
\hline \multirow{3}{*}{ counts } & \multirow{3}{*}{ Yarn properties } & \multicolumn{6}{|c|}{ Regression Coefficients } & \multirow{2}{*}{\multicolumn{2}{|c|}{$\begin{array}{c}\text { Goodness of } \\
\text { Fit }\end{array}$}} \\
\hline & & \multirow{2}{*}{ constant } & \multicolumn{5}{|c|}{ Fiber properties } & & \\
\hline & & & $\left(\mathbf{X}_{1}\right)$ & $\left(\mathbf{X}_{2}\right)$ & $\left(\mathbf{X}_{3}\right)$ & $\left(\mathbf{X}_{4}\right)$ & $\left(\mathbf{X}_{5}\right)$ & $\mathbf{R}^{2 \%}$ & $\mathbf{R}^{2}$ \\
\hline \multirow{3}{*}{80} & $\left(Y_{1}\right)$ Strength & 2483 & 55.6 & -4.45 & -3.7 & 5.45 & -11.3 & 98.6 & 98.3 \\
\hline & $\left(\mathbf{Y}_{2}\right)$ Neps & 2220 & -38.2 & 3.08 & 12.7 & 13.2 & -23.1 & 96.2 & 95.5 \\
\hline & $\left(Y_{3}\right)$ Evenness (c.v\%) & 7.97 & 0.366 & -0.0462 & -0.308 & 0.0818 & -0.033 & 96.9 & 96.3 \\
\hline \multirow{3}{*}{100} & $\left(Y_{1}\right)$ Strength & -6067 & 505 & -18.7 & -513 & 22.7 & -152 & 70.7 & 64.6 \\
\hline & $\left(\mathbf{Y}_{2}\right) \mathrm{Neps}$ & 2382 & 53 & -23.2 & -65.1 & 24.3 & -71.6 & 96.0 & 95.1 \\
\hline & $\left(\mathbf{Y}_{3}\right)$ Evenness (c.v\%) & 15.6 & $0 . \overline{-}$ & -0.0366 & 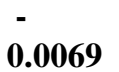 & 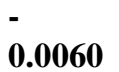 & 0.0789 & 94.8 & 93.7 \\
\hline \multirow{3}{*}{120} & (Y)Strength & 1440 & 47.0 & 5.20 & -1.5 & 1.16 & -0.85 & 96.8 & 96.1 \\
\hline & $\left(\mathbf{Y}_{2}\right) \mathrm{Neps}$ & 3106 & 99.2 & -45.0 & -87 & 35.1 & -104 & 94.7 & 93.7 \\
\hline & $\left(\mathbf{Y}_{3}\right)$ Evenness (c.v\%) & 29.7 & 0.917 & -0.273 & -0.868 & 0.323 & -0.727 & 91.6 & 89.9 \\
\hline
\end{tabular}

X1= (U.H.M), Upper half mean Length (m.m), X2= (F. stre.), Fiber strength (g/tex), X3( Mic.), Micronaire, X 4= (S.F.C), Short fiber content and $\mathrm{X} 5=(\mathrm{F} . \mathrm{d})$, Fiber diameter

When theyarn properties Y1 strength, Y2neps count and Y3evenness (c.v\%)on counts 80, 100 and 120 individually regressed on the tested fiber properties under combed ring spinning system for Extra-long stable Egyptian cotton varieties the results were as in table (3). All postulated regression models were significant and properties on counts 8 os, 1 oos and 120 s expressed as high values of $\mathrm{R}^{2}$ ranged from 82.2 to $98 \%$ the previous results proved the validity of the supposed models of regression, in addition, the clear closeness between $\mathrm{R}^{2}$ values and their corresponding adjusted $\mathrm{R}^{2}$ values gave another evidence. Accordingly, the present regression equations would be accurately applied to predict the theeyarn properties on counts 80,100 and 120 using fiber traits. The current results are in harmony with El-Hariryet al. (1990), Sawires et al. (1990) and Fares et al. (2010).
Table 4 shoed the best equation amount, coefficient of determination (R2) and rank of contribution of the studied fiber properties toyarn strength, neps and evenness (c.v\%) within each yarn counts (80, 100 and 120) over the Extra-long - Extra fine stable Egyptian cotton varieties. Concerning the Extra-long - Extra fine stable Egyptian cotton varieties, the step wise multiple linear regression indicated that the accepted limiting properties of cotton fiber that were significantly accounted for the most variation of yarn strength (Y1) on count 8oswereUpper half mean length(m.m) $\left(\mathrm{x}_{1}\right)$, fiber diameter $\left(\mathrm{x}_{5}\right)$ and micronaire $\left(\mathrm{x}_{3}\right)$ while fiber diameter $\left(\mathrm{x}_{5}\right)$, Upper half mean length $(\mathrm{m} . \mathrm{m})\left(\mathrm{x}_{1}\right)$ and short fiber content $\left(\mathrm{x}_{4}\right)$ were most important properties using the neps $\left(\mathrm{y}_{2}\right)$ on count 80 s as a dependent variable. For evenness $\left(\mathrm{y}_{3}\right)$ on count $8 \mathrm{os}$, 
only the threefiber properties of Upper half mean length (m.m) ( $\left.\mathrm{x}_{1}\right)$,fiber strength $(\mathrm{g} /$ tex $)\left(\mathrm{x}_{2}\right)$ and micronaire $\left(\mathrm{x}_{3}\right)$ were accepted using the stepwisemodel. the three proposed models were responsible for 98,95.08 and $94.5 \%$ of the total variation(expressed as $\mathrm{R}^{2} \%$ ) of yarn strength $\left(\mathrm{y}_{1}\right)$,neps $\left(\mathrm{y}_{2}\right)$ and evenness $\left(\mathrm{y}_{3}\right)$ on count $8 \mathrm{os}$ respectively. According to the previous results, it could be concluded that Upper half mean length (m.m) $\left(\mathrm{x}_{1}\right)$, fiber strength $(\mathrm{g} / \mathrm{tex})\left(\mathrm{x}_{2}\right)$ and micronaire $\left(\mathrm{x}_{3}\right)$ were the most effective fiber properties overall the twoyarn properties yarn strength $\left(\mathrm{y}_{1}\right)$, neps $\left(\mathrm{y}_{2}\right)$ on count 1 oos of Extra-long - Extra fine stable Egyptian cotton varieties $\left(\mathrm{R}^{2}=7 \mathrm{o}, 94.65 \%\right.$ respectively). The best equation of three variables effectingyarn evenness $\left(\mathrm{y}_{3}\right)$ were fiber diameter $\left(\mathrm{x}_{5}\right)$, short fiber content $\left(\mathrm{x}_{4}\right)$ and Upper half mean length (m.m) $\left(\mathrm{x}_{1}\right) \quad\left(\mathrm{R}^{2}=94.6\right)$. The best equation of four variables effectingyarn strength $\left(\mathrm{y}_{1}\right)$ on count $12 \mathrm{os}$ were Upper half mean length (m.m) $\left(\mathrm{x}_{1}\right)$, fiber strength $(\mathrm{g} /$ tex $)\left(\mathrm{x}_{2}\right)$, short fiber content $\left(\mathrm{x}_{4}\right)$ and Micronaire $\left(x_{3}\right)\left(R^{2}=91.5\right)$.the best equation of four variables effecting evenness $\left(\mathrm{y}_{3}\right)$ on count $12 \mathrm{os}$ were fiber strength $(\mathrm{g} / \mathrm{tex})\left(\mathrm{x}_{2}\right)$, Micronaire $\left(\mathrm{x}_{3}\right)$, fiber diameter $\left(\mathrm{x}_{5}\right)$ and Upper half mean length (m.m) $\left(\mathrm{x}_{1}\right)$ $\left(\mathrm{R}^{2}=88 \%\right)$. The current results are portly in accordance with those reported by Amin (1971) and sawires et al. (1990). Remarkable results were obtained from the previous result, it is that, the fiber properties which significantly contributing toyarn properties differed according to the Extra-long Extra fine stable of the used Egyptian cotton varieties Sawires et al. (1990).

Table 3. Regression Coefficient to predict fiber and yarn properties within each of three yarncounts(80, 100 and 120) in two staples of Egyptian cotton

\begin{tabular}{|c|c|c|c|c|c|c|c|c|c|}
\hline \multirow{3}{*}{ counts } & \multirow{3}{*}{ Yarn properties } & \multicolumn{6}{|c|}{ Regression Coefficients } & \multirow{2}{*}{\multicolumn{2}{|c|}{$\begin{array}{c}\text { Goodness of } \\
\text { Fit } \\
\end{array}$}} \\
\hline & & \multirow{2}{*}{ constant } & \multicolumn{5}{|c|}{ Fiber properties } & & \\
\hline & & & $\left(\mathbf{X}_{1}\right)$ & $\left(\mathbf{X}_{2}\right)$ & $\left(\mathbf{X}_{3}\right)$ & $\left(\mathrm{X}_{4}\right)$ & $\left(\mathrm{X}_{5}\right)$ & $\mathbf{R}^{2 \%}$ & $\mathbf{R}^{2}$ \\
\hline \multirow{3}{*}{80} & $\left(Y_{1}\right)$ Strength & 639 & -9.70 & 47.60 & $\mathbf{7 2 . 7 0}$ & -31.9 & 20.3 & 82.2 & 81.4 \\
\hline & $\left(\mathrm{Y}_{2}\right) \mathrm{Neps}$ & 50.0 & -0.084 & -1.73 & -1.96 & 0.862 & 1.08 & 95.7 & 95.5 \\
\hline & $\left(Y_{3}\right)$ Evenness (c.v\%) & 73.2 & -0.698 & -0.634 & -0.104 & 0.364 & -0.236 & 88.2 & 87.7 \\
\hline \multirow{3}{*}{100} & $\left(Y_{1}\right)$ Strength & 147 & 54.70 & 19.10 & 69.50 & -14.8 & 2.77 & 98.0 & 97.9 \\
\hline & $\left(\mathrm{Y}_{2}\right) \mathrm{Neps}$ & 15.3 & 0.96 & -1.30 & -2.62 & 1.08 & 0.769 & 95.7 & 95.5 \\
\hline & $\left(Y_{3}\right)$ Evenness (c.v\%) & 26.3 & 0.055 & -0.285 & -1.31 & 0.146 & 0.009 & 95.4 & 95.3 \\
\hline \multirow{3}{*}{120} & $\left(Y_{1}\right)$ Strength & 8543 & 6.30 & 29.20 & -206 & 87.90 & -163 & 90.6 & 90.2 \\
\hline & $\left(\mathrm{Y}_{2}\right) \mathrm{Neps}$ & 293 & -2.69 & -3.49 & 1.26 & 0.836 & -0384 & 96.7 & 96.6 \\
\hline & $\left(\mathrm{Y}_{3}\right)$ Evenness (c.v\%) & 54.2 & -0.967 & -0.410 & 0.736 & 0.305 & 0.153 & 93.9 & 93.6 \\
\hline
\end{tabular}

X1= (U.H.M), Upper half mean Length (m.m), X2= (F. stre.), Fiber strength (g/tex), X3( Mic.), Micronaire, X 4= (S.F.C), Short fiber content and $\mathrm{X} 5=(\mathrm{F} . \mathrm{d})$, Fiber diameter

Table 4. The best equation amount coefficient of determination $\left(\mathrm{R}^{2}\right)$ and rank of Contribution of the studied fiber properties to yarn properties within each yarn counts $(80,100$ and 120) in two staples of Egyptian cotton.

\begin{tabular}{|c|c|c|c|c|c|c|c|c|}
\hline \multirow{2}{*}{ counts } & \multirow{2}{*}{$\begin{array}{c}\text { Yarn } \\
\text { Properties }\end{array}$} & \multirow{2}{*}{ Best Equation } & \multirow{2}{*}{$\begin{array}{l}\mathbf{R}^{2} \\
\%\end{array}$} & \multicolumn{5}{|c|}{ Rank of contribution } \\
\hline & & & & first & second & third & fourth & fifth \\
\hline \multirow{3}{*}{80} & $\left(\mathrm{Y}_{1}\right)$ Strength & $\begin{array}{l}2237+65.5\left(\mathrm{x}_{1}\right)-11.07\left(\mathrm{x}_{5}\right)-5.77\left(\mathrm{x}_{2}\right)- \\
17.2\left(\mathrm{x}_{3}\right)\end{array}$ & 98.0 & $\mathrm{X} 1$ & $\mathrm{X} 3$ & $\mathrm{X} 2$ & $\mathrm{X} 4$ & $\mathrm{X} 5$ \\
\hline & \multirow{2}{*}{$\begin{array}{l}\left(\mathrm{Y}_{2}\right) \mathrm{Neps} \\
(\mathrm{Y} 3) \text { Evenness } \\
\text { (c.v\%) }\end{array}$} & $1883-21.4\left(\mathrm{x}_{5}\right)-25\left(\mathrm{x}_{1}\right)+4.5\left(\mathrm{x}_{4}\right)$ & 95.08 & $\mathrm{X} 5$ & $\mathrm{X} 1$ & $\mathrm{X} 4$ & $\mathrm{X} 2$ & $\mathrm{X} 3$ \\
\hline & & $5.369+0.445\left(\mathrm{x}_{1}\right)-0.07\left(\mathrm{x}_{2}\right)-0.388\left(\mathrm{x}_{3}\right)$ & 94.5 & $\mathrm{X} 4$ & X5 & $\mathrm{X} 1$ & $\mathrm{X} 2$ & $\mathrm{X} 3$ \\
\hline \multirow{3}{*}{100} & $\left(\mathrm{Y}_{1}\right)$ Strength & $-7091+546\left(\mathrm{x}_{1}\right)-24\left(\mathrm{x}_{2}\right)-151\left(\mathrm{x}_{5}\right)-569\left(\mathrm{x}_{3}\right)$ & 70.0 & $\mathrm{X} 1$ & $\mathrm{X} 4$ & $\mathrm{X} 3$ & $\mathrm{X} 5$ & $\mathrm{X} 2$ \\
\hline & $\left(\mathrm{Y}_{2}\right) \mathrm{Neps}$ & $\begin{array}{l}1281-70.8\left(\mathrm{x}_{5}\right)-29.1\left(\mathrm{x}_{2}\right)+97.3\left(\mathrm{x}_{1}\right)-125 \\
\left(\mathrm{x}_{3}\right)\end{array}$ & 94.65 & $\mathrm{X} 5$ & $\mathrm{X} 2$ & $\mathrm{X} 1$ & $\mathrm{X} 4$ & $\mathrm{X} 3$ \\
\hline & $\begin{array}{l}\left(\mathrm{Y}_{3}\right) \text { Evenness } \\
(\mathrm{c} . \mathrm{v} \%)\end{array}$ & $16.2+0.0594\left(\mathrm{x}_{5}\right)+0.0225\left(\mathrm{x}_{4}\right)-0.0274\left(\mathrm{x}_{1}\right)$ & 94.6 & $\mathrm{X} 5$ & $\mathrm{X} 4$ & $\mathrm{X} 1$ & $\mathrm{X} 3$ & $\mathrm{X} 2$ \\
\hline \multirow{3}{*}{120} & $\left(\mathrm{Y}_{1}\right)$ Strength & $\begin{array}{l}955.7+66.7\left(\mathrm{x}_{1}\right)+2.19\left(\mathrm{x}_{2}\right)-9.40\left(\mathrm{x}_{4}\right)-30.6 \\
\left(\mathrm{x}_{3}\right)\end{array}$ & 96.5 & $\mathrm{X} 1$ & $\mathrm{X} 2$ & $\mathrm{X} 5$ & $\mathrm{X} 3$ & $\mathrm{X} 4$ \\
\hline & \multirow{2}{*}{$\begin{array}{l}\left(\mathrm{Y}_{2}\right) \mathrm{Neps} \\
(\mathrm{Y} 3) \text { Evenness } \\
(\mathrm{c.v} \%)\end{array}$} & $977-129.4-47.3\left(x_{2}\right)+211\left(x_{1}\right)-294$ & 91.5 & $\mathrm{X} 5$ & $\mathrm{X} 2$ & $\mathrm{X} 1$ & $\mathrm{X} 4$ & $\mathrm{X} 3$ \\
\hline & & $\begin{array}{l}15.12-0.351\left(\mathrm{x}_{2}\right)-1.66\left(\mathrm{x}_{3}\right)-0.717\left(\mathrm{x}_{5}\right) \\
+1.51\left(\mathrm{x}_{1}\right)\end{array}$ & 88.3 & $\mathrm{X} 2$ & $\mathrm{X} 5$ & $\mathrm{X} 1$ & $\mathrm{X} 4$ & $\mathrm{X} 3$ \\
\hline
\end{tabular}

X1=(U.H.M), Upper half mean Length (m.m), X2= (F. stre.), Fiber strength (g/tex), X3( Mic.), Micronaire, X 4= (S.F.C), Short fiber content and $\mathrm{X} 5=(\mathrm{F} . \mathrm{d})$, Fiber diameter

Table 5 showed the prediction the best equation amount, coefficient of determination $\left(\mathrm{R}^{2}\right)$ and rank of contribution of the studied fiber properties toyarn strength, neps and evenness (c.v \%) 
within each yarn counts $(80,100$ and 120) over the Extra-long stable Egyptian cotton varieties. Concerning the Extra-long stable Egyptian cotton varieties, the step wise multiple linear regression indicated that the accepted limiting properties of cotton fiber that were significantly accounted for the most variation of yarn strength $\left(\mathrm{y}_{1}\right)$,neps $\left(\mathrm{y}_{2}\right)$ on count 8 os were fiber diameter $\left(\mathrm{x}_{5}\right)$, short fiber content $\left(\mathrm{x}_{4}\right)$,Micronaire $\quad\left(\mathrm{x}_{3}\right)$ and fiber strength $(\mathrm{g} /$ tex $)\left(\mathrm{x}_{2}\right)$ while fiber strength $(\mathrm{g} /$ tex $)\left(\mathrm{x}_{2}\right)$,short fiber content $\left(\mathrm{x}_{4}\right)$ and Upper half mean length $(\mathrm{m} . \mathrm{m})\left(\mathrm{x}_{1}\right)$ were important properties usingevenness $\left(\mathrm{y}_{3}\right)$ on count 8 os as a dependent variable. The threemodels on count 80 accounted for 82, 95.7 and $87.1 \%$ expressed as $\mathrm{R}^{2}$ of the total variation of yarn strength $\left(\mathrm{y}_{1}\right)$, neps $\left(\mathrm{y}_{2}\right)$ andevenness $\left(\mathrm{y}_{3}\right)$ respectively. The technique of stepwise regression accepted Micronaire $\left(\mathrm{x}_{3}\right)$, fiber strength $(\mathrm{g} / \mathrm{tex})\left(\mathrm{x}_{2}\right)$, short fiber content $\left(\mathrm{x}_{4}\right)$ and Upper half mean length (m.m) $\left(\mathrm{x}_{1}\right)$ as the fiber properties that they had greatest influenceon yarn strength $\left(\mathrm{y}_{1}\right)$ count 1 loos.the trails of fiber diameter $\left(\mathrm{x}_{5}\right)$, short fiber content $\left(\mathrm{x}_{4}\right)$ and fiber strength $(\mathrm{g} /$ tex $)\left(\mathrm{x}_{2}\right)$ contributing to the neps $\left(\mathrm{y}_{2}\right)$ on count 1 oos variation. It may be reported that both Micronaire $\left(\mathrm{x}_{3}\right)$, short fiber content $\left(\mathrm{x}_{4}\right)$ and fiber strength $(\mathrm{g} / \mathrm{tex})\left(\mathrm{x}_{2}\right)$ were the important fiber properties to predict yarn evenness $\left(\mathrm{y}_{3}\right)$ on count 1oos using Extra-long stable Egyptian cotton varieties. The values of $97.9,94.15$ and 95.4 (expressed as $\mathrm{R}^{2} \%$ ) were the ability of the three proposed models to predict the variation of the three tested yarn properties on count 100 s $\left(\mathrm{y}_{1}, \mathrm{y}_{2}\right.$ and $\left.\mathrm{y}_{3}\right)$ respectively. the best equation of three variables effectingyarn strength $\left(\mathrm{y}_{1}\right)$ on count 120 were short fiber content $\left(\mathrm{x}_{4}\right)$, Micronaire $\left(\mathrm{x}_{3}\right)$ and fiber diameter $\left(\mathrm{x}_{5}\right)$. According to previous results, it could be concluded that fiber diameter $\left(\mathrm{x}_{5}\right)$, Upper half mean length (m.m) $\left(\mathrm{x}_{1}\right)$, short fiber content $\left(\mathrm{x}_{4}\right)$ and fiber strength $(\mathrm{g} /$ tex $)\left(\mathrm{x}_{2}\right)$ were the most effective fiber properties overall the twoyarn properties neps $\mathrm{y}_{2}$, evenness $y_{3}$ on count 12 os for the Extra-long stable Egyptian cotton varieties. The values of 90.5,96.6 and $93.3 \%$ expressed (as $\mathrm{R}^{2 \%}$ ) were the ability of the thee proposed models to predict thevariation of three tested yarn properties on count $120\left(\mathrm{y}_{1}, \mathrm{y}_{2}\right.$ and $\left.\mathrm{y}_{3}\right)$ respectively. Similar trends of results wereobtained by Sief (1984), Hegabet al. (1985), El-Hariryet al. (1990), Sawires et al. (1990) and Fares et al. (2010).

Table 5 . The best equation amount coefficient of determination $\left(\mathrm{R}^{2}\right)$ and rank of Contribution of the studied fiber properties to yarn properties within each yarn counts $(80,100$ and 120) in two staples of Egyptian cotton.

\begin{tabular}{|c|c|c|c|c|c|c|c|c|}
\hline \multirow{2}{*}{ counts } & \multirow{2}{*}{$\begin{array}{c}\text { Yarn } \\
\text { properties }\end{array}$} & \multirow{2}{*}{ Best Equation } & \multirow{2}{*}{$\mathbf{R}^{2 \%}$} & \multicolumn{5}{|c|}{ Rank of contribution } \\
\hline & & & & first & secon & third & ourth & fifth \\
\hline \multirow{3}{*}{80} & $\left(\mathrm{Y}_{1}\right)$ Strength & $412.7+19.9\left(\mathrm{x}_{5}\right)-33\left(\mathrm{x}_{4}\right)+55.3\left(\mathrm{x}_{3}\right)+46.0\left(\mathrm{x}_{2}\right)$ & 82.0 & $\mathrm{X} 5$ & $\mathrm{X} 2$ & $\mathrm{X} 4$ & $\mathrm{X} 3$ & $\mathrm{X} 1$ \\
\hline & $\left(\mathrm{Y}_{2}\right) \mathrm{Neps}$ & $\begin{array}{l}48.04+1.074\left(\mathrm{x}_{5}\right)+0.853\left(\mathrm{x}_{4}\right)-1.74\left(\mathrm{x}_{2}\right)-2.12 \\
\left(\mathrm{x}_{3}\right)\end{array}$ & 95.7 & $\mathrm{X} 5$ & $\mathrm{X} 2$ & $\mathrm{X} 3$ & $\mathrm{X} 1$ & $\mathrm{X} 4$ \\
\hline & $\begin{array}{l}\text { (Y3)Evenness } \\
(\text { c.v\%) }\end{array}$ & $49.04-0.355\left(\mathrm{x}_{2}\right) .+0.371\left(\mathrm{x}_{4}\right) \quad-0.688\left(\mathrm{x}_{1}\right)$ & 87.1 & $\mathrm{X} 5$ & $\mathrm{X} 3$ & $\mathrm{X} 1$ & $\mathrm{X} 2$ & $\mathrm{X} 4$ \\
\hline \multirow{3}{*}{100} & $\left(\mathrm{Y}_{1}\right)$ Strength & $416.5+67.4\left(\mathrm{x}_{3}\right)+15.78\left(\mathrm{x}_{2}\right) 14.3\left(\mathrm{x}_{4}\right)+55.1\left(\mathrm{x}_{1}\right)$ & 97.9 & $\mathrm{X} 3$ & $\mathrm{X} 1$ & $\mathrm{X} 5$ & $\mathrm{X} 2$ & $\mathrm{X} 4$ \\
\hline & $\left(\mathrm{Y}_{2}\right) \mathrm{Neps}$ & $-8.189+1.258\left(\mathrm{x}_{5}\right)+1.058\left(\mathrm{x}_{4}\right)+0.73\left(\mathrm{x}_{2}\right)$ & 94.15 & $\mathrm{X} 5$ & $\mathrm{X} 2$ & $\mathrm{X} 4$ & $\mathrm{X} 3$ & $\mathrm{X} 1$ \\
\hline & $\begin{array}{l}\text { (Y3)Evenness } \\
(\text { c.v\%) }\end{array}$ & $28.723-1.221\left(\mathrm{x}_{3}\right)+0.154\left(\mathrm{x}_{4}\right)-0.294\left(\mathrm{x}_{2}\right)$ & 95.4 & $\mathrm{X} 3$ & $\mathrm{X} 1$ & $\mathrm{X} 5$ & $\mathrm{X} 2$ & $\mathrm{X} 4$ \\
\hline \multirow{3}{*}{120} & $\left(\mathrm{Y}_{1}\right)$ Strength & $11134+92.9\left(\mathrm{x}_{4}\right)-206\left(\mathrm{x}_{3}\right) 186.1\left(\mathrm{x}_{5}\right)$ & 90.5 & $\mathrm{X} 2$ & $\mathrm{X} 5$ & $\mathrm{X} 1$ & $\mathrm{X} 4$ & $\mathrm{X} 3$ \\
\hline & $\left(\mathrm{Y}_{2}\right) \mathrm{Neps}$ & $\begin{array}{l}283.28-0.457\left(\mathrm{x}_{5}\right)- \\
2.05\left(\mathrm{x}_{1}\right) 3.59\left(\mathrm{x}_{2}\right)+0.780\left(\mathrm{x}_{4}\right)\end{array}$ & 96.6 & X5 & $\mathrm{X} 2$ & $\mathrm{X} 1$ & $\mathrm{X} 3$ & $\mathrm{X} 4$ \\
\hline & $\begin{array}{l}\text { (Y3)Evenness } \\
\text { (c.v\%) }\end{array}$ & $\begin{array}{l}48.339+0.111\left(\mathrm{x}_{5}\right)-0.59\left(\mathrm{x}_{1}\right)+0.272\left(\mathrm{x}_{4}\right)- \\
0.470\left(\mathrm{x}_{2}\right)\end{array}$ & 93.3 & X5 & $\mathrm{X} 2$ & $\mathrm{X} 1$ & $\mathrm{X} 3$ & $\mathrm{X} 4$ \\
\hline
\end{tabular}

X1= (U.H.M), Upper half mean Length (m.m), X2= (F. stre.), Fiber strength (g/tex), X3( Mic.), Micronaire, X 4= (S.F.C), Short fiber content and $\mathrm{X} 5=(\mathrm{F} . \mathrm{d})$, Fiber diameter

Finally, the current investigation stated the following conclusions or remarks:-

1- The results of the supposed regression models differed according to the category of the used cotton variety and also the kind of the applying spinning system.

2- The current results helps the spinner to predict the spinning performance using the available fiber properties as well as choosing cotton that are best suited to the manufacture of the end products.

3- Statistically, goodness of fit was satisfied for all regression models under the present investigation.
4- The residuals content $\left(1-\mathrm{R}^{2}\right)$ may be attributed to three reasons being the committed errors during measuring fiber and yarn properties, some considerable fiber properties were not into account and/ or unknown variation (random error).

5- The stepwise regression procedure determined the minimum number of fiber characters that are accounted for the most variation of various yarn properties which save the time and effort.

6- The stepwise regression procedure determined the minimum number of fiber characters that are 
accounted for themost variation of various yarn properties which save the time and effort

\section{References}

A.S.T.M. (American Society for Testing and Materials) 1967, 1986 .Standards of textile materials.

Abd El-Fattah, M. Kh. (1988). Study of the relative importance of cotton fiber properties on fiber and yarn strength. Ph. D. Thesis fac. Agric. Ain Shams Univ. Cairo, Egypt.

Abd-Salam, M.E. 1999 . The Egyptian cotton production quality and Elkalemapress .Box No16/o5/99

Aboul-Fadl, S. M. 1976 . Cotton fiber structure and its relation to quality -element.Ph. D. Thesis, Fac. Agric., Cairo Univ.

Ahmed, H. S.A and Hassan, A.A 2016 .The relationship between fiber properties and neps formation in yarn for some Egyptian cotton varietiesAnnals of Agric. Sci., Moshtohor.54 (3).

Ahmed, M. S. ; Kama, M. M. 1 and Yousef, A. E. 1984 .The contribution of micronaire reading, fiber length and fiber stiffness to yarn nippiness. Agric. Res. Rev. Egypt, 62, 259-266.

Amin, A. Z. 1971 . Studies on fiber properties and spinning quality of cotton. Ph. D. Thesis, Fac. Univ. of Alex., Egypt.

Balasubramanian, N. and R. L. N. Lyengar 1961 .Study of the relation of yarn irregularitywith fiber properties and its effect on yarn strength.Ind. Text. J., 71:561-567.

Draper, N. R. and R. Smith 1981 .Applied regression analysis.John Wiley and sons, Inc.NewYork.7o4 pp.

El-Ghawas, M. 1., Abd El-Salam M. E. and Garawain M. S. E. 1978 .Study of the relationships of yarn unevenness and nippiness with fiber properties Agric. Res. Rev., Egypt. 56: 33-41.

EL-Hariry, S. H. M., Mansour F. S., Sawires E. M. S. and .Seif M. G 1990 .The relative contribution of fiber properties to yarn physical properties in Giza 77 Egyptian cotton variety byusing stepwise regression analysis.Agric. Res. Review, 68 (6): 1287-1297.

Fares, W. M., Islam S. K. A., Hussein K. M. and Hassan A.A 2010 An application of modern statistical approach to estimate a technological value of sameEgyptian cotton Varieties. The sixth Inter. Conf. of Sustain Agric. and Develop. 27-29 December, 43-56.

Fiori, L. A., Brown J. J. and Sonds J. E. 1954 .Effect of cotton fiber strength on singleyarn properties and on processing behavior. Text. Res. J. 24: 503-507.

Garawain, M. S. 1971 . Study of the effect of some cotton fiber properties on yarn evenness. M. Sc. Thesis, Fac. Agric. Ain-Shams Univ. Egypt.
Grosberg, P. (1956).Correlation between mean fiber length and yarn irregularity. J. Text. Inst. 47: T179-T18o.

Hager, M. A., Hassan A. A. and Fares W. M. 2011 Some Statistical Relationships Models to Predict Yarn Properties Using Fiber Properties for Two Categories of Egyptian cotton Varieties under Two Spinning Systems. Agricultural Research Journal; Suez Canal University

Hassan A.A. and Ibrahim A.E. 2018 .Using some fiber properties to predict strength and regularity of carded yarns for some Egyptian cotton varieties. Middle East Journal of Applied Sciences V. (8) P. (1256-1263).

Hegab, A. A. T., Youssef A. E. and Abdellah M. E. 1985 .Prediction of yarn strength of Egyptian cotton. Agric. Res. Rev. Egypt. 63: 155-162.

Krifa M. and Ethridge D. 2003 . Compact ring spun yarns: an examination of some productivity issues. Textile Topics. 2: 2-8.

Krifa M. and Ethridge D. 2006 .Compact spinning effect on cotton yarn quality: Interactions with Fiber Characteristics. Textile Research Journal (TRJ), 76(5): 388-399.

Leitgeb, D. L. and Wakeham H. 1956 .Cotton quality and fiber properties effect of fiber fineness. Tex. Res. J. 26: 343-352.

Louis, G. L.,Fiori L. A. and Leitz L. A. 1968 .Relationships among fiber properties, yarn properties and end breakage for medium staple cotton. Tex. Bull. 94(6): 43-48.

Louis, G. L., Fiori L. A. andSads J. E. 1961 .Blending cotton differing in fiber bundle break elongation. 1- Effect on properties of combed singleyarns. Text. Res. J. 31: 43-48.

Mansour, F. S. 1984 .Effect of physical characteristics of cotton on yarn strength and irregularity of Egyptian cottonPh. D. Thesis, Fac. Agric. Ain Shams Univ.

Marth, G. T., H. E. Arthur and E. E. Berkley 1952 .Fiber fineness neps in web and yarn appearancegrades. Tex. Res. J., 22:561-566.

Nawar, M. T.; Ghorab,.M. A. andSeif,M.G 1990 . A study of structural propertiesoffibers different Length groups and its relativerelation to Tensile strength in some imported cultivars cultivated in Egypt. Proc. Int. comf .st.\&comp. Sci. Res and DEM,139-153

Phillip, P. C. 1957 . Study on the irregularity of theproducts of cotton spinning. Ind. Text. J., 62: 734-737.

RaoofM., A. 1995 Studies on some technological properties of new Egyptian Cotton Ph. .D .thesis ,facofagric, Moshtohor, zagaziguniv, Egypt,18op.

Rusca, R. A. 197o .Cotton fiber properties. Cot. Gr. Rev., 47:2o5-216.

Sagbaş A. and Erol R. 2004 .Process optimization of yarn neps formation, yarn strength and cost for staple - yarn.YA/EM' YöneylemAraştırması 
/EndüstriMühendisliği -Proc. UlusalKongresi, 15 18 Haziran Gaziantep - Adana.

Sallouma, B. M., M. R. Abd El-Mohsen and M. A. Shaker 1973 .Fiber fineness in relation to fiber and yarn properties. Cotton Exp. Assoc., 61: 3344.

Sawires, E. M. S., Seif M. G., EL-Hariry S. H. M. and Mansour F. S. 1990 .The relative contribution of fiber properties to yarn mechanical properties in Giza 77 Egyptian cotton variety byusing stepwise regression analysis. Agric. Res. Review, 68 (6): 1299-1308.
Sief, M. G. 1984 . Spinningperformance as affected byyarn count, Twist factor and fiber properties in someEgyptian cotton varieties. Ph. D. Thesis, Fac. Agric., Ain Shams Univ., Egypt.

Strumiło L. J., Cyniak D., Czekalski J. and Jackowski T. 2007 . Quality of cotton yarns spun using ring-, compact-, and rotor-spinning machines as a function of selected spinning process parameters. Fibers\& Textiles in Eastern Europe January / March 2007, 15, No. 1 (60): 24 30.Text. J. 49: 26-30.Wiley and Sons, Inc, New York.

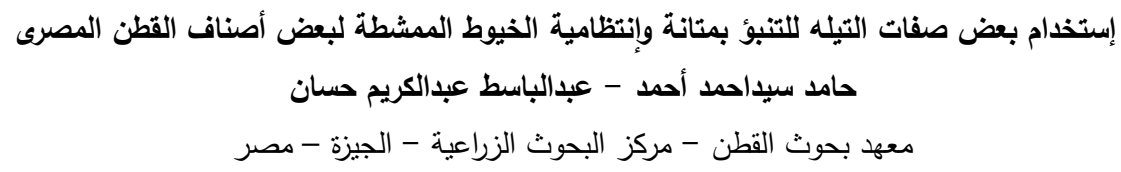

يهدف هذا البحث لدراسة مدى مساهمة صفات التيلة في صفات الخيوط الممشطة (متانة الثلة، العقد، انتظامية الخيط)عند نمره 80 ،100، 120 لاصناف القطن المصري (فائق الطول فائق النعومة و فائق الطول) تحت نظام الغزل الحلقي ـ وقد اشتملت الأصناف فائقة الطول والنعومة علي صنف (جيزة 45، جيزة 87 و جيزة 93) بينما كانت الأصناف فائقة الطول هي (جيزة 92، جيزة 88، جيزة 70 ) حيث نم استخدام عينات من المحصول التجاري لعام 2016

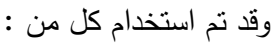
R² - $\quad$ - $\quad$ تحليل الانحدار المتعدد

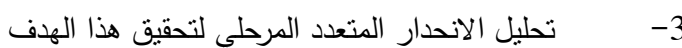
وقد أوضحت النتائج أن صفات التيلة الأكثر إسهاماً في صفات الخيط الممشط تختلف من نمرة لآخرى ومن فئة إلي أخري كما اختلفت ايضا درجة الارتباطRم من نمرة لآخرى ومن فئة إلي أخري

وبوجه عام أثنالت النتائج الى ان طول اطول الثعيرات كانت هي الأكثر تأثثراً في صفات الخيوط المشطة (متانة الثلة، العقد، انتظامية الخيط)عند نمر نمره 80 ،100، 120 انجليزى وذلك لأصناف القطن فائق الطول والنعومة والاصناف فائقة الطول بينما كانت صفات منانة التيلة، والميكرونير هي الأهم في نوقع صفات الخيط المشطة على نمرة 120 انجليزى لأصناف القطن فائقة الطول الناعمة. قطر الثعره هي أكثر صفات التبلة إسهاماً في صفات الخيوط المشطة على نمرة 120 انجليزى لأصناف القطن فائقة الطول في

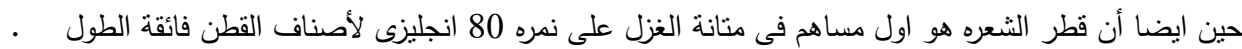

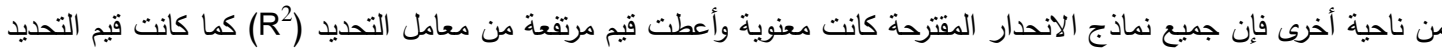

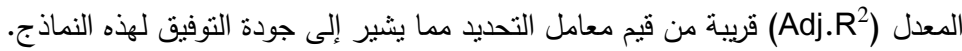

